\title{
Hybrid therapy with locoregional steroid injection and polyglycolic acid sheets to prevent stricture after esophageal endoscopic submucosal dissection
}

Authors

Institution
Yasuaki Nagami, Masatsugu Shiba, Kazunari Tominaga, Masaki Ominami, Shusei Fukunaga, Satoshi Sugimori, Fumio Tanaka, Noriko Kamata, Tetsuya Tanigawa, Hirokazu Yamagami, Toshio Watanabe, Yasuhiro Fujiwara, Tetsuo Arakawa

Department of Gastroenterology, Osaka City University Graduate School of Medicine, Osaka, Japan submitted 23. March 2016 accepted after revision 13. June 2016

\section{Bibliography}

Dol http://dx.doi.org/ 10.1055/s-0042-111906 Published online: 25.8.2016 Endoscopy International Open 2016; 04: E1017-E1022 (c) Georg Thieme Verlag KG Stuttgart · New York E-ISSN 2196-9736

\section{Corresponding author} Kazunari Tominaga, MD, PhD Department of

Gastroenterology

Osaka City University Graduate School of Medicine

1-4-3

Asahimachi

Abeno-ku

Osaka

545-8585

Japan

Fax: +81-6-66453813

tomy@med.osaka-cu.ac.jp
Background and study aim: The incidence of stricture formation caused by endoscopic submucosal dissection (ESD) for widespread lesions is high, and stricture formation can reduce quality of life. We evaluated the prophylactic efficacy of hybrid therapy using a locoregional steroid injection and polyglycolic acid (PGA) sheets with fibrin glue to prevent stricture formation after esophageal ESD in high risk patients in whom we predicted stricture formation would be difficult to prevent with a single prophylactic steroid injection. Methods: Ten patients who underwent esophageal ESD were enrolled (entire-circumference: $\mathrm{n}=6$; sub-circumference, more than $5 / 6$ of the circumference: $n=4)$. A single locoregional steroid injection and PGA sheets with fibrin glue were used after ESD. We evaluated the inci-

\section{Introduction \\ $\nabla$}

Endoscopic submucosal dissection (ESD) can use an en bloc resection procedure for superficial esophageal neoplasia, regardless of tumor size $[1,2]$. However, stricture formation after ESD has been reported in $90 \%$ of patients with a mucosal defect greater than $75 \%$ of the circumference [3], and stricture formation often leads to poor quality of life. In addition, frequent endoscopic balloon dilation (EBD) procedures are required to treat stricture formation, and repeated EBD increases medical costs and the risk of adverse events [3]. Locoregional steroid injection and oral steroid administration are useful for preventing post-ESD stricture formation [4-9]. However, oral steroids must be administered for at least 3 weeks, and it is difficult to treat some patients such as those with diabetes mellitus [7-11]. Recent case reports indicated that polyglycolic acid (PGA) sheets, a biodegradable suture material, affixed with fibrin glue safely prevented esophageal stricture formation [12,13]. However, the prevention rate was not sufficiently high, and the occur- dence of stricture formation, the number of endoscopic balloon dilation (EBD) procedures needed to treat the stricture formation, and adverse events of the therapy.

Results: Esophageal stricture formation occurred in $50.0 \%$ of patients $(5 / 10)$ (median EBD sessions 0.5 , range $0-16$ ). Subanalysis showed that stricture formation occurred in $37.5 \%$ of patients $(3 / 8)$ excluded the lesions located near a previous scar from ESD or surgical anastomosis site (median EBD sessions 0, range 0-4).

Conclusion: Hybrid therapy using a locoregional steroid injection and PGA sheets with fibrin glue may have the potential to prevent esophageal stricture formation after esophageal ESD in high risk patients.

rence of stricture was higher than that after steroid therapy. In addition, a single prophylactic treatment with steroid injection or PGA sheets is ineffective for an entire-circumferential resection. Several cases of stricture formation after prophylactic steroid therapies have been reported [4-6, 11]. This higher rate of stricture formation might be due to baseline expansion of the circumference of the mucosal defect into an entire-circumference defect.

We investigated the prophylactic efficacy of hybrid therapy using a steroid injection and PGA sheets with fibrin glue to prevent stricture formation after esophageal ESD in high risk patients in whom we predicted stricture formation would be difficult to prevent with a single prophylactic steroid injection. 


\begin{tabular}{|c|c|c|}
\hline \multicolumn{3}{|c|}{$\begin{array}{l}\text { ESD for esophageal neoplasia } \\
\text { between November } 2013 \text { and June } 2015 \\
184 \text { cases }\end{array}$} \\
\hline \multicolumn{2}{|c|}{$\begin{array}{l}\text { The high-risk population } \\
\text { (1) With lesions of circumferential }>5 / 6 \\
\text { (2) With lesions circumferential }>1 / 2 \text { with RT } \\
\text { (3) With lesions circumferential }>1 / 2 \text { with scar }\end{array}$} & $\begin{array}{l}10 \text { cases } \\
6 \text { cases } \\
3 \text { cases } \\
2 \text { cases }\end{array}$ \\
\hline \multicolumn{3}{|c|}{$\checkmark$ ESD } \\
\hline \multicolumn{2}{|c|}{$\begin{array}{l}\text { Circumferential mucosal defect } \geq 5 / 6 \\
\text { (1) Circumferential mucosal defect } \geq 11 / 12 \\
\text { (2) Circumferential mucosal defect } \geq 5 / 6 \text { with RT } \\
\text { (3) Circumferential mucosal defect } \geq 5 / 6 \text { with scar }\end{array}$} & $\begin{array}{l}10 \text { cases } \\
6 \text { cases } \\
3 \text { cases } \\
2 \text { cases }\end{array}$ \\
\hline & \multicolumn{2}{|l|}{ Hybrid therapy } \\
\hline \multicolumn{3}{|c|}{ Underwent analysis 10 cases } \\
\hline \multirow[t]{2}{*}{$\begin{array}{l}\text { Entire circumference } 6 \text { case } \\
\text { (1) } 5 \text { cases } \\
\text { (2) } 1 \text { case * } \\
\text { (3) } 1 \text { case }^{*}\end{array}$} & $\begin{array}{l}\text { Circumference } \geq 5 / 6 \\
\text { (1) } 1 \text { case } \\
\text { (2) } 2 \text { cases } \\
\text { (3) } 1 \text { case }\end{array}$ & 4 cases \\
\hline & Exclude 2 cases of ( 3 ) & \\
\hline \multicolumn{3}{|c|}{ Subanalysis 8 cases } \\
\hline $\begin{array}{l}\text { Entire circumference } 5 \text { cases } \\
\text { (1) } 5 \text { cases } \\
\text { (2) } 0 \text { case }\end{array}$ & $\begin{array}{l}\text { Circumference } \geq 5 / 6 \\
\text { (1) } 1 \text { case } \\
\text { (2) } 2 \text { cases }\end{array}$ & 3 cases \\
\hline
\end{tabular}

Fig. 1 Study flow chart. * One patient had both previous radiotherapy and lesion upon previous ESD scar. RT, previous radiotherapy.

\section{Materials and methods}

$\nabla$

\section{Patients}

This retrospective case series was performed at Osaka City University. Between November 2013 and June 2015, 184 patients with 297 lesions from superficial esophageal neoplasias were treated with ESD in our hospital ( $\bullet$ Fig. 1). All patients provided written informed consent. The high risk population included the following: (1) patients who had single or multiple adjacent lesions and superficial esophageal neoplasms over $5 / 6(83.3 \%)$ of the esophageal circumference, which were predicted to be resected with a mucosal defect greater than or equal to $11 / 12$ (91.7\%) of the esophageal circumference after ESD; patients who had single or multiple adjacent lesions and superficial esophageal neoplasms over $1 / 2(50.0 \%)$ of the esophageal circumference, which were predicted to be resected with a mucosal defect greater than $3 / 4(75.0 \%)$ of the esophageal circumference after ESD; (2) patients who had previously received radiotherapy for esophageal cancer; and (3) patients with lesions located near a previous scar from ESD or a surgical anastomosis site. Ten patients with a mucosal defect greater than or equal to $5 / 6$ of the esophageal circumference who received hybrid therapy with a steroid injection and PGA sheets with fibrin glue were analyzed. The main outcome measure was the incidence of stricture formation. Secondary outcomes were the number of EBDs needed to treat stricture formation and the occurrence of adverse events. The ethics committee of the Osaka City University Graduate School of Medicine approved this study (approval no.: 3340).

\section{ESD procedure}

A single-channel upper gastrointestinal endoscope (GIF-Q260J; Olympus, Tokyo, Japan) and a standard electrosurgical generator (VIO300D; ERBE Elektromedizin GmbH, Tübingen, Germany) were used. After delineating the tumor margins using iodine staining ( $\bullet$ Fig. 2a), marking dots were placed using a bipolar needle-knife (B knife; XEMEX Co., Tokyo, Japan) or a monopolar needle-knife (Flush knife, DK2618JN; Fujifilm Medical, Tokyo, Japan). A hyaluronic acid solution was injected into the submucosal layer. After circumferential mucosal incision, submucosal dissection was performed ( $\bullet$ Fig. 2 b) $[2,5]$. The lesion's size and mucosal defect were measured as a proportion of the esophageal circumference that was removed, based on its division into 12 equal parts ( $\bullet$ Table 1$)$ [12].

\section{Locoregional steroid injection and PGA sheet development}

A single session of triamcinolone acetonide (Kenacort; BristolMyers Squibb, Tokyo, Japan) was injected into the residual submucosal layer of the resected region immediately after ESD ( $\nabla$ Video 1 ); $80 \mathrm{mg}$ of triamcinolone acetonide was injected regardless of the size of the resected specimens, followed by 40 sequential injections of $0.5 \mathrm{~mL}$ ( $2 \mathrm{mg}$ ) of triamcinolone acetonide ( $\bullet$ Fig.2c) [5]. Subsequently, pieces of PGA sheet (Neoveil; Gunze, Kyoto, Japan) were inserted to fill the mucosal defect using biopsy forceps without through-the-scope channel $(\bullet$ Fig. 2d) [12]. Finally, we applied fibrinogen and thrombin solutions of fibrin glue (Beriplast P Combi-Set; CSL Behring Pharma, Tokyo, Japan) to the PGA sheets ( $\bullet$ Fig. 2 e). In the final four patients, we used the clip-and-pull method [13]. Endoscopy was performed 2 days after ESD to confirm attachment of the PGA sheets and assess for adverse events $[12,13]$. Then an oral diet was resumed.

\section{Follow-up and stricture formation}

Endoscopic evaluation of stricture formation was performed every 4 weeks after the procedure. When an ESD ulceration had not healed into a scar at 8 weeks, endoscopic examination was also performed 4 weeks later ( $\bullet$ Fig.2f). Esophageal stricture was considered present when a standard endoscope for the upper gastrointestinal tract, $9.2 \mathrm{~mm}$ in diameter, could not pass through the treatment site. When the patient presented with a chief complaint of dysphagia to semi-solid foods (dysphagia score 2 ) before examination, endoscopic evaluation was performed at that time. EBD was repeatedly performed when a stricture persisted either endoscopically or symptomatically.

\section{Results \\ $\nabla$}

\section{Clinicopathological characteristics}

Hybrid therapy was performed in (1) six patients who had superficial esophageal neoplasms greater than or equal to $11 / 12$ of the circumferential defect, (2) two patients who had superficial esophageal neoplasms greater than or equal to $5 / 6$ of the circumferential defect with a history of radiotherapy, (3) one patient who had a superficial esophageal neoplasm $5 / 6$ of the circumferential defect located at a surgical anastomosis site, and (2)+(3) one patient who had an entire circumferential defect located on a previous scar from ESD and a history of radiotherapy ( $\bullet$ Fig. 1$)$. The clinicopathological characteristics of the 10 patients (nine men and one woman; median age 71.0 years) are shown in - Table 1. Two patients underwent en bloc resection of two adjacent lesions. Six patients had an entire circumferential defect and four had a sub-circumferential defect greater than or equal to $5 / 6$ of the esophageal circumference. All neoplasias were resect- 

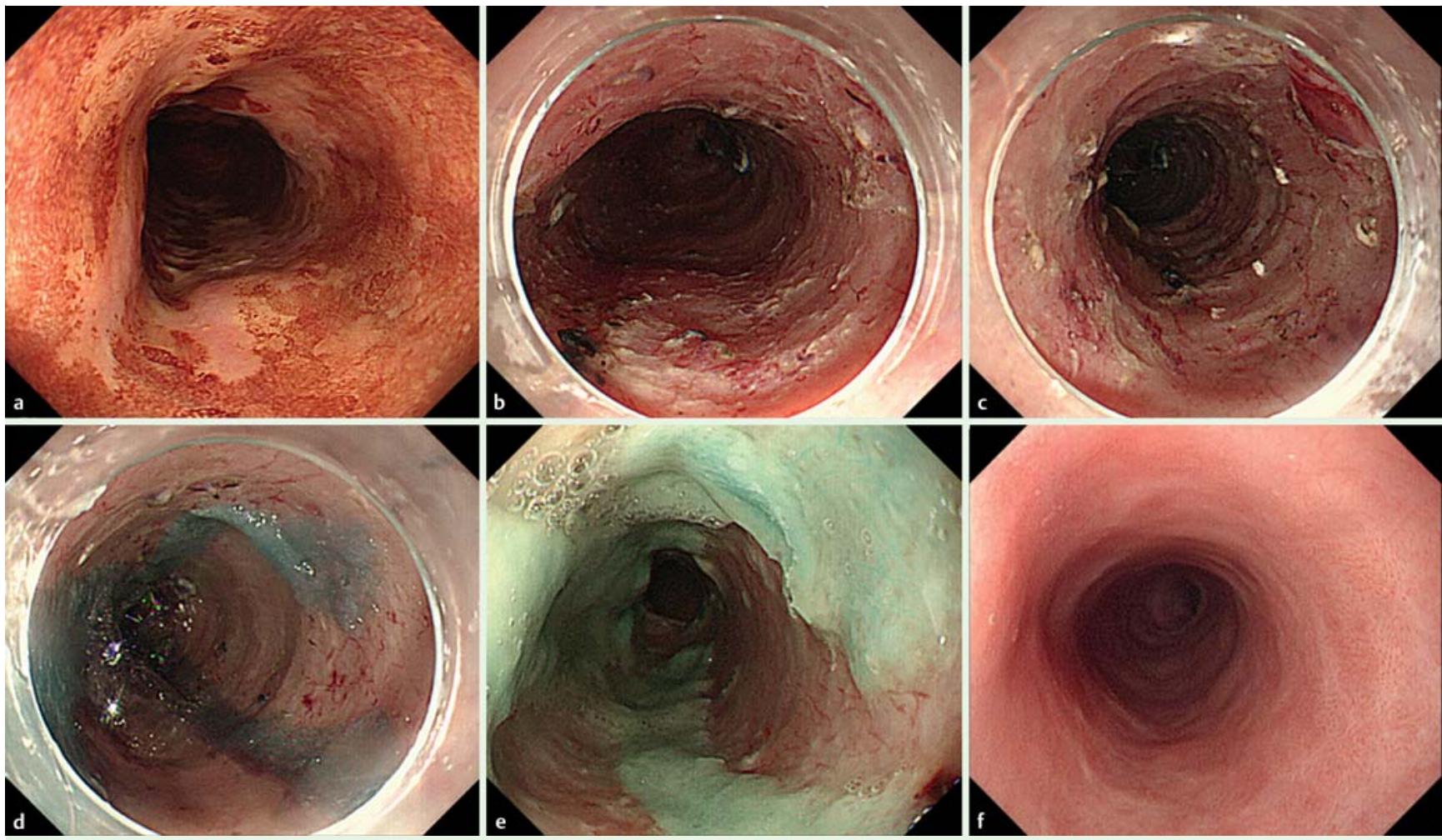

Fig.2 Endoscopic appearance of hybrid therapy using locoregional steroid injection and polyglycolic acid (PGA) sheets with fibrin glue. Subtotal circumferential esophageal neoplasia was observed by chromoendoscopy with iodine staining (a), and circumferential endoscopic submucosal dissection (ESD) was performed (b). A steroid solution was carefully injected into the residual superficial submucosal layer (c). Pieces of PGA sheets were used to fill the mucosal defect using biopsy forceps. Subsequently, fibrin glue was applied to the PGA sheets (d). The PGA sheets remained in place upon observation on day 1 post-ESD (e). Endoscopic view showing that the ESD-induced ulceration improved without stricture and that a conventional upper gastrointestinal endoscope could be passed through the treatment site $(\mathbf{f})$.

ed en bloc with negative margins. Patient 2 underwent additional esophagectomy without stricture formation over 81 days. Patient 3 had stricture formation before another operation was performed, and it did not resolve within 68 days. Patient 6 underwent additional chemotherapy 85 days later, and patient 4 received additional chemoradiotherapy 160 days later.

\section{Stricture formation}

Esophageal stricture formation occurred in five of 10 patients $(50.0 \%)($ Table 1$)$. Stricture formation occurred in four of six (66.7\%) patients with entire circumferential defects and one of four $(25.0 \%)$ patients with sub-circumferential mucosal defects. The median duration between ESD and stricture confirmation of the first EBD was 49 days (range 32 - 65).

The number of EBD sessions required to treat stricture formation was a median of 0.5 sessions (range $0-16$ ); two patients with refractory stricture still require EBD. No adverse events occurred associated with the prophylactic therapy, but one perforation associated with EBD was observed in patient 4 after chemoradiotherapy.

\section{Subanalysis}

We performed a subanalysis of eight patients excluding the patients with lesions located near a previous scar from ESD or surgical anastomosis site. Patient 9 had esophageal intramural pseudodiverticulosis, which is a rare cause of esophageal stricture, and he underwent ESD for esophageal cancer with 2/3 of a circumferential mucosal defect, chemoradiotherapy for local recurrence with an entire circumference, and entire circumferen- tial salvage ESD for second recurrence with a sub-circumferential defect. Additionally, he had a moderate esophageal stricture. Patient 10 had a history of esophagectomy for esophageal cancer, and then he underwent total gastrectomy for gastric tube cancer, reconstruction of esophago-jejunal anastomosis, and ESD for three recurrent esophageal tumors that developed at the anastomosis site with mild stricture.

Esophageal stricture formation occurred in three of eight patients $(37.5 \%$ ) with a median of 0 EBD sessions (range $0-4$ ) ( $\bullet$ Table 1). All patients with stricture formation had undergone entire-circumferential resection $(60.0 \%, 3 / 5$ patients), whereas no stricture formation occurred in the three patients with subcircumferential mucosal defects.

Among six patients (five with entire circumferential defects, one with sub-circumferential defect) without a history of radiotherapy, stricture formation occurred in three (50.0\%) (median EBD sessions 0.5 , range $0-3$ ).

\section{Discussion}

Hybrid therapy using a locoregional steroid injection and PGA sheets with fibrin glue may have the potential to reduce stricture formation caused by ESD for superficial esophageal neoplasms in high risk patients in whom we predicted stricture formation would be difficult to prevent with a single steroid injection. Additionally, this combination therapy requires a low number of EBD sessions, even in patients with stricture formation. 


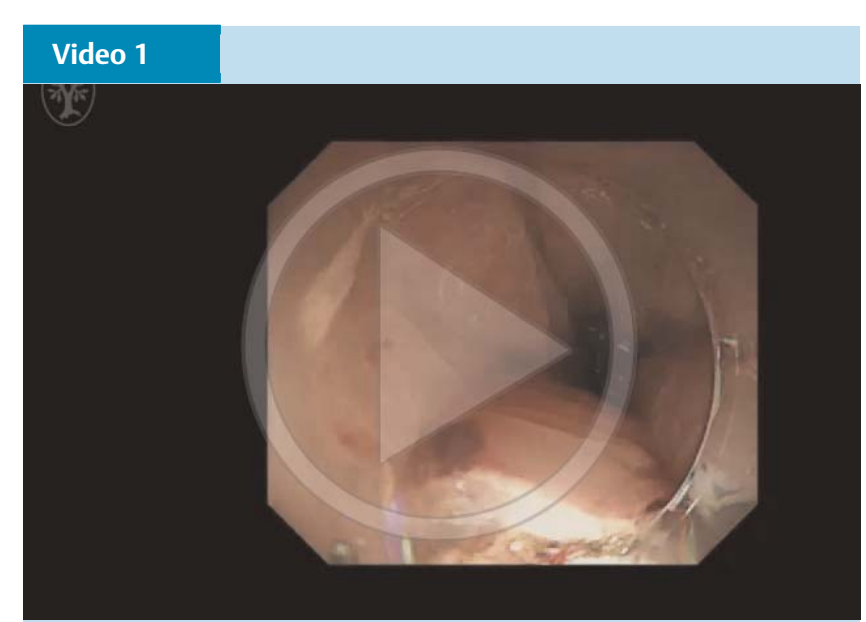

After circumferential endoscopic submucosal dissection (ESD), a steroid solution was carefully injected into the residual superficial submucosal layer. Then, pieces of polyglycolic acid (PGA) sheets were used to fill the mucosal defect using biopsy forceps. Subsequently, fibrin glue was applied to the PGA sheets. Online content including video sequences viewable at: http://dx.doi. org/10.1055/s-0042-111906

Locoregional steroid injection reduces the incidence of stricture formation by approximately $10-19 \%$ with minimum adverse events in patients with more than $3 / 4$ circumferential mucosal defect $[4,5]$. However, an entire circumferential mucosal defect after ESD was difficult to prevent with several prophylactic therapies [6,7,9-11,14]. Additionally, a tumor greater than $3 / 4$ of the circumference was reported to be a risk factor for refractory stricture despite steroid injection [14]. Furthermore, a history of radiotherapy and tumor recurrence on a previous scar from ESD and esophagectomy site were considered risk factors for stricture, and these cases with over 3/4 of a circumferential defect were considered to be a higher risk for stricture despite steroid injection. Therefore, we enrolled these high risk patients, because they require effective prophylactic therapy.

Overall, half of the patients had strictures, whereas $37.5 \%$ of patients had strictures in the subanalysis. Thus, the therapy may not be effective for tumor recurrence on a previous scar from ESD and esophagectomy site with over a 5/6 circumferential mucosal defect. However, strictures were prevented in two patients with a history of radiotherapy. Because a history of radiotherapy was not reported as a risk factor for refractory stricture [14], it may be acceptable to include these cases in the analysis. Stricture did not occur in eight patients with sub-circumferential defects even though we enrolled patients with larger circumferential defects compared to those in a previous report. As only $18.2 \%$ of strictures occurred in another setting of hybrid therapy [15], this treatment may have the potential to prevent stricture in patients with sub-circumferential defects. Fewer EBD sessions were needed to treat stricture formation in our study compared to previous studies (range $0-1.7$ ) ( $\bullet$ Table 2) [4-7,11-13].

The higher incidence of stricture $(60 \%)$ was caused by a higher proportion of cases with entire circumferential defects. Several prophylactic therapies did not prevent stricture formation in most of these cases ( Table 2) $[9-11,14]$. Since tissue contraction with centripetal movement of the landmarks in the esophageal mucosa is a normal part of the healing process of the ulcer [16], residual mucosa and sub-circumferential resection play an important role in preventing stricture. In addition, 3.3-13.8 or 

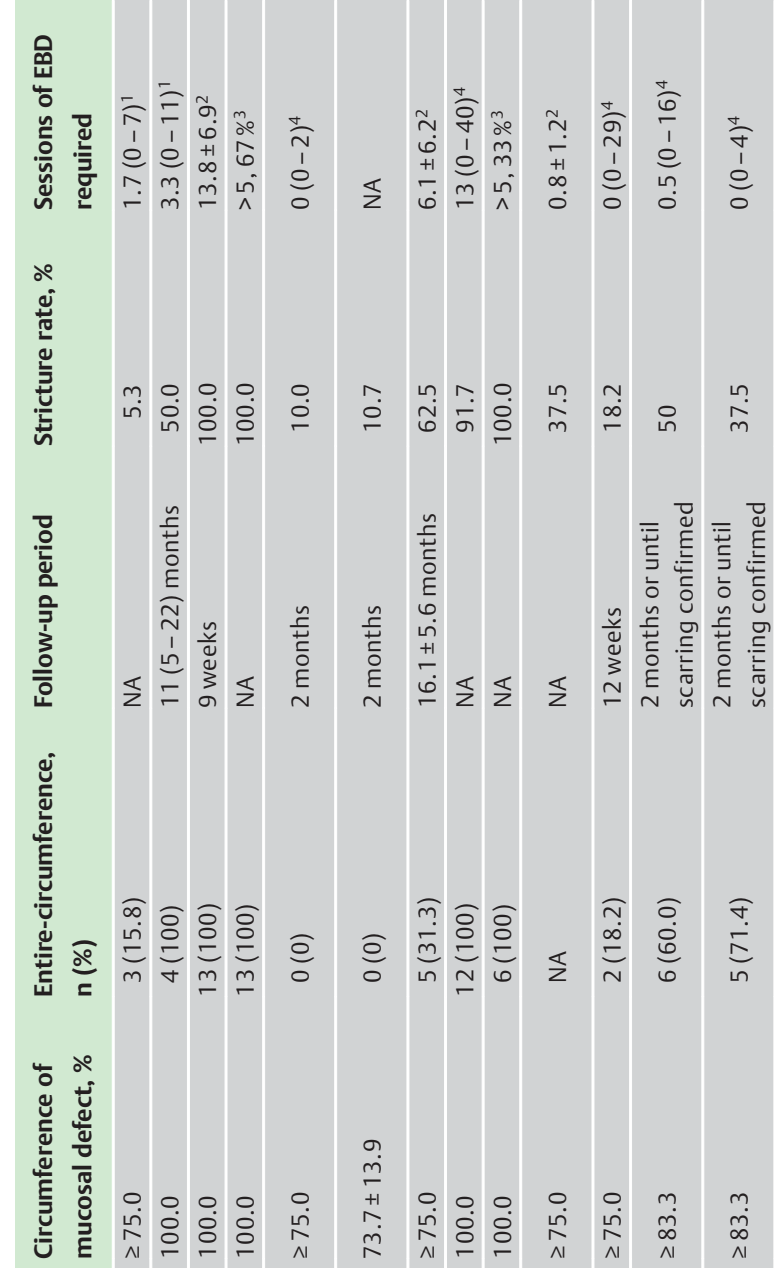

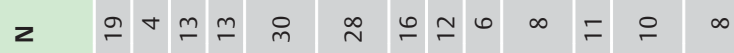

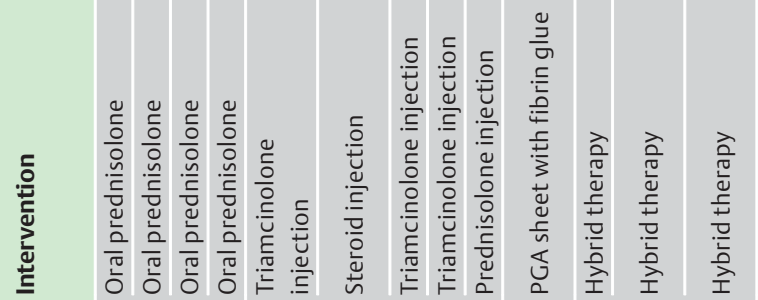

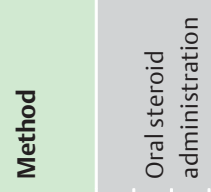

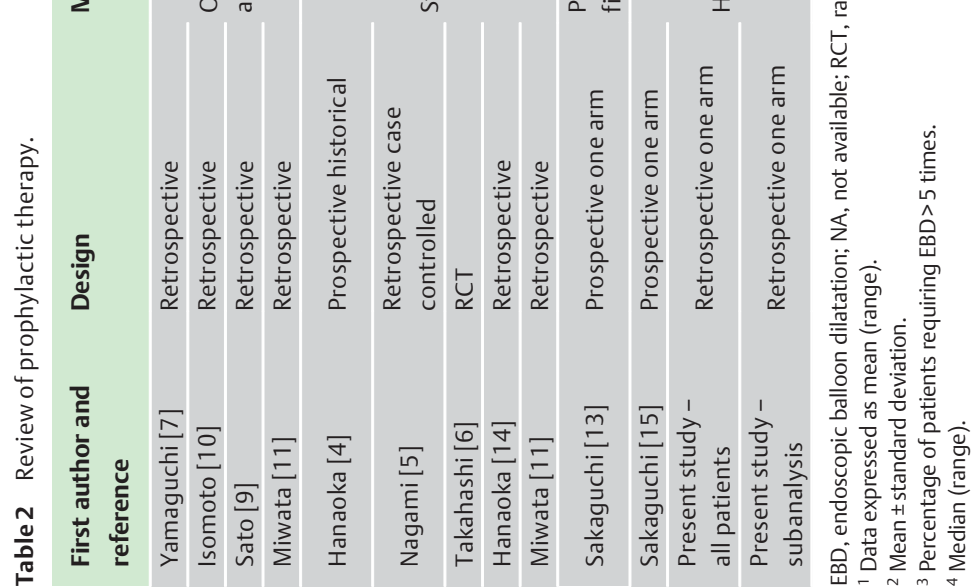


10.4 EBD sessions were reported after oral or locoregional steroid therapy, respectively [6,9-11], whereas a median of 1 (range 0 4 ) was required in the present study. Miwata et al. showed that a longitudinal mucosal defect $\geq 5 \mathrm{~cm}$ was a risk factor for refractory stricture requiring more than five EBD sessions after an entire circumferential resection [11]. However, fewer EBD sessions were required to treat stricture formation, even in those with mucosal defects $\geq 5 \mathrm{~cm}$ long ( Table 1 ). Our therapeutic methods may be useful for those with entire-circumferential mucosal defects.

Intralesional corticosteroid injection is thought to prevent stricture formation by inhibiting fibrosis and inflammation [5]. Although the detailed mechanisms remain unknown, Iizuka et al. speculated that PGA sheets with fibrin glue shield the surface of the ESD ulceration and protect it from buccal secretions and food, thus inhibiting inflammation, because PGA sheets with fibrin glue prevent scar formation and contraction after glossectomy [12]. Hybrid therapy using steroid injection and PGA sheets likely has additive or synergistic effects. We hope that the detailed mechanisms will be elucidated in basic studies.

Our study has several limitations. First, this was a retrospective case series with a small sample size. Large, prospective, randomized studies are needed. Second, the delivery of the PGA sheets was technically difficult and lengthy, thus the development of a new, easier delivery system is needed. Third, the inclusion of additional therapy may have affected our outcomes. However, the incidence of stricture formation may be accurate; scarring without stricture was confirmed in patient 9 before esophagectomy, and stricture was confirmed in the other patients before esophagectomy or chemoradiotherapy. Yet, this could have affected the number of EBD sessions.

Hybrid therapy using a locoregional steroid injection and PGA sheets with fibrin glue may have the potential to prevent esophageal stricture after ESD for superficial esophageal neoplasms in high risk patients.

Competing interests: Yes; Tetsuo Arakawa serves on advisory committees for Otsuka Pharmaceutical Co. Ltd and Eisai Co. Ltd. Yasuhiro Fujiwara serves on an advisory committee for Ono Pharmaceutical Co. Ltd and Toshio Watanabe serves on an advisory committee for Eisai Co. Ltd. The other authors declare that they have no conflict of interest.

\section{References}

1 Kim JS, Kim BW, Shin IS. Efficacy and safety of endoscopic submucosal dissection for superficial squamous esophageal neoplasia: a meta-analysis. Dig Dis Sci 2014; 59: 1862 - 1869
2 Nagami Y, Machida H, Shiba $M$ et al. Clinical efficacy of endoscopic submucosal dissection for adenocarcinomas of the esophagogastric junction. Endosc Int Open 2014; 2: E15-20

3 Ono S, Fujishiro M, Niimi K et al. Predictors of postoperative stricture after esophageal endoscopic submucosal dissection for superficial squamous cell neoplasms. Endoscopy 2009; 41: 661-665

4 Hanaoka $N$, Ishihara $R$, Takeuchi $Y$ et al. Intralesional steroid injection to prevent stricture after endoscopic submucosal dissection for esophageal cancer: a controlled prospective study. Endoscopy 2012; 44: $1007-1011$

5 Nagami $Y$, Shiba $M$, Tominaga $K$ et al. Locoregional steroid injection prevents stricture formation after endoscopic submucosal dissection for esophageal cancer: a propensity score matching analysis. Surg Endosc 2016; 30: 1441 - 1449

6 Takahashi H, Arimura Y, Okahara S et al. A randomized controlled trial of endoscopic steroid injection for prophylaxis of esophageal stenoses after extensive endoscopic submucosal dissection. BMC Gastroenterol 2015; $15: 1$

7 Yamaguchi N, Isomoto H, Nakayama T et al. Usefulness of oral prednisolone in the treatment of esophageal stricture after endoscopic submucosal dissection for superficial esophageal squamous cell carcinoma. Gastrointest Endosc 2011; 73: 1115-1121

8 Kataoka M, Anzai S, Shirasaki T et al. Efficacy of short period, low dose oral prednisolone for the prevention of stricture after circumferential endoscopic submucosal dissection (ESD) for esophageal cancer. Endosc Int Open 2015; 3: E113-117

9 Sato H, Inoue H, Kobayashi Y et al. Control of severe strictures after circumferential endoscopic submucosal dissection for esophageal carcinoma: oral steroid therapy with balloon dilation or balloon dilation alone. Gastrointest Endosc 2013; 78: 250-257

10 Isomoto H, Yamaguchi N, Nakayama T et al. Management of esophageal stricture after complete circular endoscopic submucosal dissection for superficial esophageal squamous cell carcinoma. BMC Gastroenterol 2011; $11: 46$

11 Miwata T, Oka S, Tanaka S et al. Risk factors for esophageal stenosis after entire circumferential endoscopic submucosal dissection for superficial esophageal squamous cell carcinoma. Surg Endosc 2015. DOI: $10.1007 /$ s00464-015-4719-3

12 Iizuka T, Kikuchi D, Yamada A et al. Polyglycolic acid sheet application to prevent esophageal stricture after endoscopic submucosal dissection for esophageal squamous cell carcinoma. Endoscopy 2015; 47: $341-344$

13 Sakaguchi Y, Tsuji Y, Ono $S$ et al. Polyglycolic acid sheets with fibrin glue can prevent esophageal stricture after endoscopic submucosal dissection. Endoscopy 2015; 47: 336 - 340

14 Hanaoka $N$, Ishihara $R$, Uedo $N$ et al. Refractory strictures despite steroid injection after esophageal endoscopic resection. Endosc Int Open 2016; 4: E354-359

15 Sakaguchi $Y$, Tsuji $Y$, Fujishiro $M$ et al. Triamcinolone injection and shielding with polyglycolic acid sheets and fibrin glue for postoperative stricture prevention after esophageal endoscopic resection: a pilot study. Am J Gastroenterol 2016; 111: 581 - 583

16 Ohki T, Yamato M, Ota $M$ et al. Prevention of esophageal stricture after endoscopic submucosal dissection using tissue-engineered cell sheets. Gastroenterology 2012; 143: 581-588 